\title{
Review: Plant Growth Hormone Cytokinins Control the Crop Seed Yield
}

\author{
Norimoto Murai \\ Department of Plant Pathology and Crop Physiology, Louisiana State University and LSU Agricultural Center, \\ Baton Rouge, USA \\ Email: nmurai@lsu.edu
}

Received 15 May 2014; revised 13 June 2014; accepted 27 June 2014

Copyright (C) 2014 by author and Scientific Research Publishing Inc. This work is licensed under the Creative Commons Attribution International License (CC BY). http://creativecommons.org/licenses/by/4.0/ (c) (i) Open Access

\begin{abstract}
This review chronicles the development of the cytokinin research during the last 30 years. Cytokinin and auxin are the two major plant growth hormones that control virtually all aspects of growth and development in higher plants. The pathways for cytokinin biosynthesis and metabolism have been characterized by the identification of isopentenyl pyrophosphate transferase, cytokinin oxidases, cytokinin hydroxylase, zeatin cis-/trans-isomerase, cytokinin phosphoribosyl hydrolases, cytokinin-specific riboside phosphorylase, and others enzymes. Loss-of function mutant phenotypes of cytokinin degradation/activating enzymes indicate the regulation of concentration and spatial distribution of bio-active cytokinin plays a pivotal role in the increase in panicle size, in the numbers of floral organs, and eventually in seed yield. One of the most fundamental questions in the cytokinin field is one concerning the prevalence of cis-zeatin in monocotyledonous crops (rice and maize) and in dicotyledonous legumes (pea, chickpea) and potato/sweet potato. A hypothesis is that cis-zeatin is synthesized by the cis-specific hydroxylation of the terminal methyl group of $\mathrm{N}^{6}$-isopentenyl side chain of $\mathrm{N}^{6}$-isopentenyl adenosine (i6Ado) or of their mono-, di-, or tri-phosphates catalyzed by the cis-specific hydroxylase. A second potential pathway is the isomerization of trans-zeatin to cis-zeatin by zeatin cis-/trans-isomerase. A second fundamental question to be addressed is the physiological role of cis-zeatin. Some have argued for a special function of cis-zeatin to account for the prevalence of the cis-zeatin in the plant kingdom from algae to higher plants.
\end{abstract}

\section{Keywords}

Cytokinins, Biosynthesis and Metabolism, cis-and trans-Zeatin, Cytokinin Oxidase

\section{Introduction: Cytokinins Control Shoot Growth and Differentiation}

Cytokinin and auxin are the two major plant growth hormones that control virtually all aspects of growth and 
development in higher plants [1]. Skoog and Miller demonstrated the chemical control of plant development in 1957 by their classical experiment of organ regeneration of tobacco tissues cultured in the chemically defined media [2]. The higher concentration ratio of cytokinin to auxin promotes the shoot development, the equivalent ratio of cytokinin to auxin enhances the proliferation of non-differentiated calli, and the lower ratio of cytokinin to auxin supports the root development. Cytokinin plays a positive regulatory role in shoot development, and a negative regulatory role in root development [3] [4]. Active cytokinins are required in the proliferation of undifferentiated meristematic cells in the shoot apical meristem. Cytokinins are probably involved in the control of the maintenance of undifferentiated cells as a promoter of cell division, as well as in the identity of the shoot meristem. In roots, cytokinins control the rate of meristematic cell differentiation [5]. Cytokinin translocates acropetally via xylem and systemically via the phloem.

Two homeodomain transcription factors, KNOTTED1-like (KNOX) and WUSCHEL (WUS) play important roles in controlling the stem cell activities at the shoot apical meristem and repressing differentiation of cells [6][8]. WUS promotes the expression of CLAVATA 3 (CLV3) restricting the domain of WUS transcription. WUS expression is enhanced by AHK4/WOODENLEG receptors for cytokinin sensitivity. WUS also represses the type-A RESPONSE REGULATOR genes, the primary target of cytokinin signal transduction. KNOX suppresses a gibberellin (GA) biosynthesis gene for GA20-ox, and activates cytokinin biosynthesis genes for Isopentenyl Pyrophosphate Transferase (IPT) maintaining a high concentration ratio of CK/GA in a spatially controlled manner. OSH1, a rice homologues of KNOX protein, induces the expression of OsIPT2 and OsIPT3 and represses other six OsIPT genes [9]. In Arabidopsis, KNOX activates AtIPT7 gene.

The architecture of rice inflorescence (panicle) is determined by the spatial and temporal control of meristem phase change from the vegetative shoot meristem to reproductive floral meristem, and second phase change from indeterminate branch meristem to determinate spikelet meristem (flower or floret) [7]. Later the spikelet meristem specification, more branching and more grains are produced in rice panicle. A gene for ABERRANT PANICLE ORGANIZTION1 and 2 (APO1 and 2) promote branch meristem identity and inhibit spikelet meristem identity [10] [11]. PANICLE PHYTOMER 2 (PAP2) is the only positive regulator of spikelet meristem identity [12]. PAP2 suppresses rice RICE CENTROLADIALLIS 1 (RCN1), ortholog of Arabidopsis TERMINAL FLOWER 1 (TFL1) [13].

Mobile microRNAs, miRNA394, miRNA165/166 and miRNA172 are involved in maintaining the position of domains within the shoot meristem, and restricting the stem cell identity to the three outer cell layers. miRNA394 represses the F-Box protein LEAF CURING RESPONSIVENESS (LCR) which promotes leaf morphogenesis and modifies auxin response. miRNA 165/166 represses class II homeodomain basic leucine zipper (HD-ZIP) transcription factors terminating the primary shoot meristem activity. miRNA172 represses the floral meristem identity gene APETALA2 which is required for floral meristem termination [7] [8].

\section{Biosynthesis and Metabolism of Cytokinins}

The chemical structures of major cytokinins are as follows:

$\begin{array}{ll}\text { i }^{6} \text { Ade; } & \text { 6-(3-methyl-2-butenylamino)purine } \\ \text { trans-zeatin; } & \text { 6-(4-hydroxy-3-methyl-trans-2-butenylamino)purine } \\ \text { cis-zeatin; } & \text { 6-(4-hydroxy-3-methyl-cis-2-butenylamino)purine } \\ \text { dihydrozeatin; } & \text { 6-(4-hydroxy-3-methyl-butylamino)purine }\end{array}$

(a) The Stereochemical Isomers of Zeatin, trans- and cis-Zeatin

Cytokinin molecular species could be counted up to 26 molecules theoretically. There are four different types of cytokinin, including cis- and trans-zeatin, dihydrozeatin, and $\mathrm{i}^{6} \mathrm{Ade}$. There are seven different modifications of cytokinin molecules; in the adenine purine ring at $\mathrm{N}^{9}$-position ribosides, mono-, di-, and tri-phosphate ribonucleotides, and at $\mathrm{N}^{7}$ - and $\mathrm{N}^{9}$-positions glucosides; and in the isopetenyl side chain, O-glucoside [14]-[17].

The sterochemistry of zeatin has been one of central subjects of discussion in cytokinin research. First, trans-zeatin was the first naturally-occurring cytokinin isolated from maize endosperm in 1963. It was considered the predominant cytokinin as a free base, riboside, ribonucleotides or glucosides. cis-Zeatin was thought to be a modified base occurring exclusively as a constituent of transfer RNA molecules [18] [19] and released as free forms after tRNA degradation [20] [21]. However, this notion has been challenged and appeared to be overturned based on the accumulating evidence during the last 10 years. Cis-zeatin has been shown to be more abundant than trans-zeatin in sweet potato [22], potato [23], chickpea [24], pea [25] [26], maize [27]-[29], and 
rice [17]. The most recent systematic survey of plant kingdom from algae to higher plants demonstrated that ciszeatin is as the ubiquitous major cytokinin as trans-zeatin [30]. Cis-Zeatin was shown to associate with developmental stages of limited growth (seeds, senescent leaves), and trans-zeatin with active growth stages in Arabidopsis thaliana [30].

Second, trans-zeatin has been found to be a 100-fold more active cytokinin than cis-zeatin using callus growth promotion activity in tobacco bioassay [31], and 30-fold more active in Phaseolus lunatus L. var. Kingston [32]. However, the activities of trans- and cis-zeatin were nearly the same using callus growth promotion activity in Phaseolus vulgaris L. var. Great Northern [32], and using pea embryo length promotion activity at the heart-shape stage [25]. Using the growth inhibition of primary root length, trans-zeatin was 10-fold more effective than cis-zeatin in Arabidopsis, but cis-zeatin is more effective than trans-zeatin in rice [33]. Some argued for a special function of cis-zeatin to account for the prevalence of the cis-zeatin. The cis- and trans-zeatin along with $\mathrm{i}^{6}$ Ade were found to promote in yeast to a similar degree the activity of maize histidine kinase 1 and 3a (ZmHK1 and 3), while no cytokinin responsiveness was detected in ZmHK2 and 3b [29]. In Arabidopsis thaliana, trans-zeatin and $\mathrm{i}^{6}$ Ade interacted with AHK4, but not cis-zeatin [34]. Trans-Zeatin was more effective than cis-zeatin in increasing mRNA level of rice response regulator genes OsPR1, 2, 4, 6 and 9/10, but not OsPR3 [33]. Cis-Zeatin was a preferred substrate to trans-zeatin for O-glycosylation reaction by the cis-zeatinO-glucosyltransferase in rice [33]. The over-expression of the cis-zeatin-O-glucosyltransferase in maize demonstrated the abnormal phenotype including the growth retardation and tassel seed formation, suggesting the proper physiological function of cis-zeatin [35].

Third, cis-zeatin is most likely to be synthesized as substrates from free adenine bases, adenosine or its ribonucleotides, instead of degradation products of tRNAs [19]-[21]. However, no evidence was found for the enzyme reaction that would hydroxylate in the cis-configuration the terminal methyl moiety of $\mathrm{N}^{6}$-isopentenyl side chain of $i^{6}$ Ade, $i^{6}$ Ado or its ribotides [36]. The isomerization from cis- to trans-zeatin has been catalyzed by the zeatin cis-/trans-isomerase in bean [37]. About one tenth of cis-zeatin was converted to trans-zeatin when the maize culture was incubated with $1 \mu \mathrm{M}$ of cis-zeatin for $20 \mathrm{~min}$, suggesting the activity of the zeatin cis-/transisomerase in maze culture [29]. Alternatively, cis-zeatin might have promoted the production of trans-zeatin via the hydroxylation of $\mathrm{N}^{6}$-isopenteny side chain or other unknown reaction.

Sakakibara and his group recently have developed a high-throughput procedure to determine quantitatively the content of major plant growth hormones (cytokinin, auxin, gibberellin, abscisic acid) by using ultra-performance liquid chromatography (UPLC) coupled with a tandem quadrupole mass spectrometry (qMS-MS) equipped with an electrospray interface (ESI) (UPLC-ESI-qMs/MS) [17]. The amounts (pmol per g FW) of 17 cytokinin molecular species in rice were quantified in 13 separate organ parts, flower, panicle branch, flag leaves (tip, middle, basal), laminar joint, leaf sheath, and first, second and third nodes and internodes of japonica rice cultivar Nippon-bare at the flowering stage. Cis-Zeatin cytokinins were most abundant cytokinins ranging from $60.8 \%$ to $87.0 \%$ of all cytokinin species combined in all organs tested except for the tip region of flag leaves (36.0\%). N- and O-glucosides of cytokinins were the dominant form of cytokinin molecules ranging from $62.8 \%$ to $92.7 \%$ of all cytokinin molecules extracted. $\mathrm{N}^{9}$-glucoside cytokinins were more abundant than the $\mathrm{N}^{7}$ glucosides. The cis-zeatin O-glucoside was the single most predominant cytokinin identified among the 17 cytokinin molecular species examined. Thus, the biosynthetic pathway of cis-zeatin is the fundamental research question to be elucidated in rice, maize, legume and other crop plants.

(b) Enzymes for Cytokinin Biosynthesis and Metabolism

Cytokinin is synthesized by the attachment of the isopentenyl moiety of isopentenyl pyrophosphate to the $\mathrm{N}^{6}$-position of adenine molecule by the enzyme Isopentenyl Pyrophosphate Transferase (IPT). The adenine substrate of the IPT reaction could be an adenine nucleotide of transfer RNA, or an adenine molecule of ATP, ADP, or AMP [38] [39]. There are eight IPT cDNAs [38] and nine putative IPT genes reported in the Arabidopsis genome [39]. IPT2 is the only IPT gene that would use the tRNA as the substrate for the transfer reaction. The remaining seven IPT genes use the ATP, ADP or AMP as the substrate of the transfer reaction. IPT1, 5 and 8 are expressed in the plastids [40]. The remaining four enzymes localize in the cytoplasm. The spatial pattern of gene expression differs according to the IPT genes: IPT1 (plastid-specific, found in xylem precursor cell files in root, leaf axils, ovules, and immature seeds, controlled by $\mathrm{NO}_{3}$ ), IPT3 (phloem tissues), IPT4 (immature seeds with higher expression in the chalazail endosperm), IPT5 (plastid-specific, dominant in lateral root primordial, columella root caps, fruit abscission zones), IPT6 (siliques), IPT7 (phloem, the endodermis of the root elongation zones, trichomes of young leaves and in pollen tubes), and IPT8 (plastid-specific, immature seeds with higher 
expression in the chalazail endosperm). There are eight IPT genes in rice and maize phylogenetically characterized with the Arabidopsis genes [9] [41].

Trans-Zeatin is synthesized by the trans-specific hydroxylation of isopentenyl side chains of $\mathrm{N}^{6}$-isopentenyl adenosines ( $\left.\mathrm{i}^{6} \mathrm{Ado}\right)$ and their ribonucleotides. Cytochrome P450 monooxygenases CYP735A1 and A2 hydroxylate in a stereospecific orientation the terminal methyl group of isopenteny side chain of i ${ }^{6}$ Ado of 5 '-mono-, di-, and tri-phosphates to trans-zeatin ribosides of 5'-mono-, di-, and tri-phosphates [36]. No cis-zeatin was detectable in the media of yeast expressing these Arabidopsis enzymes.

Dihydrozeatin was converted from trans-zeatin by the zeatin reductase isolated from bean [42]. Cis-Zeatin, zeatin riboside and $i^{6}$ Ado are not substrate of the enzyme. The enzyme requires NADPH for the reaction. Zeatin cis-/trans-isomerase was also isolated from immature bean seeds [37]. The enzyme requires light, FAD or FMN. The interconversion reaction favors the formation of trans-zeatin.

Cytokinin phosphoribosyl hydrolase (LOG) catalyzes the reaction to convert cytokinin riboside 5'-monophosphate to cytokinin base and ribose 5'-monophosphate [43]. This single-step cytokinin activation process enables to increase the concentration of bioactive free cytokinin from less active cytokinin nucleoside 5'-monophosphates. The substrate specificity of LOG is for cytokinin 5'-mono-phophates, but not for 5'-di- nor tri-phosphate. LOG is a cytokinin-specific enzyme and does not discriminate among cytokinin type, either transor cis-zeatin, dihydrozeatin or i ${ }^{6}$ Ade.

Cytokinin-specific riboside phosphorylase (CKP) mediates a reversible reaction between the bioactive form of cytokinin base (with ribose-1-phosphate) and the less bioactive form of cytokininriboside (with inorganic phosphate) [44]. The enzyme has over 100-fold more affinity to trans-zeatin (20 nM K $\mathrm{M}_{\mathrm{M}}$ for zeatin, vs $3.3 \mathrm{mM}$ for adenine). The enzyme StCKP can increase the concentration of active cytokinin base in apical shoot meristem thus promoting shoot elongation while StCKP can reduce the concentration of active cytokinin by facilitating the formation of less active storage-form cytokinin riboside in dormant tissue.

Cytokinin oxidase/dehydrogenase (CKX) sits at the exit point of cytokinin metabolism that would inactivate the biological activity of cytokinin [45]. The CKX enzyme selectively recognizes the double bond of $\mathrm{N}^{6}$-isopentenyl side chain and cleaves the side chain from the $\mathrm{N}^{6}$-position of adenine molecule of active cytokinin species. The substrate preference of the enzyme is for $i^{6}$ Ade and $i^{6}$ Ado and less for trans- and cis-zeatin. The enzyme does not react with dihydrozeatin maintaining the biological activity of this cytokinin intact.

Thus, the activity of IPT and cytokinin oxidase could be important in maintaining the macro-concentration of cytokinin in the plant bodies. The activity of cytokinin phosphoribosyl hydrolase (LOG) and riboside phosphorylase (CKP) could be critical in the developmental control of micro-concentration of bioactive cytokinin in targeted cells of shoot apical meristem, flower organs or potato stolon. The activity of shoot apical meristem could be controlled by the fine-tuning of concentrations and of the spatial distribution of bioactive cytokinins by the cytokinin-activating enzymes such as LOG and CKP.

\section{Function of Cytokinin: Loss-of-Function Mutant Analysis}

\section{(a) Cytokinin Oxidase in Arabidopsis thaliana}

Cytokinin oxidase/dehydrogenase (CKX) cleaves the isopentenyl side chain from the $\mathrm{N}^{6}$-position of adenine molecule of bioactive cytokinin species [45] [46]. The genome of Arabidopsis thaliana has seven CKX genes while the rice genome has 11 CKX genes [47]. The AtCKX3 gene is most similar to the gene OsCKX2 based on phylogenetic analysis of Arabidopsis and rice proteins, while the AtCKX5 gene is most similar to the gene OsCK5. The expression of AtCKX3 is localized to the central WUSCHEL domain of the reproductive shoot meristems, while the expression of AtCKX 5 distributed over the entire meristem region.

The phenotype of loss-of-function mutants of cytokinin oxidase was analyzed by Schmulling and his group [3]-[5] [47]-[49]. T-DNA insertion to AtCKX3 and 5 genes essentially reduced the expression of these genes to none detectable level by RT-PCR. Double mutation of ckx3 ckx5 resulted in more flowers due to the formation of a larger inflorescence meristem (more cell number), larger size of flowers, $40 \%$ more siliques, and twice as many ovules per gynoecium than wild type [47]. The double mutation of ckx3 ckx5 caused 55\% increase in total seed yield, i.e. 110 seeds compared with 65 seeds in wild type [47]. Thus, cytokinin appears to increase the activity of reproductive meristem in Arabidopsis.

The cytokinin contents in inflorescence of double mutants ckx3 ckx5 increased by 4-fold for trans-zeatin and its riboside, and by 6-fold for trans-zeatin ribonucleotides which are predominant cytokinin species accounting 
for $84 \%$ of cytokinins [47]. Thus, the increased cytokinin content caused 55\% increase in seed yield, highlighting the relevance of sink strength (its capacity to make use of the fixed carbon) without increasing the strength of the $\mathrm{CO} 2$-fixing source as a seed yield factor.

(b) Cytokinin Oxidase Os CKX2 in Rice: QTL Analysis

The major agronomic traits of crop plants in general consist of a number of genes, not a single gene, with continuous phenotypic variation within the agronomic trait. The major traits were characterized as quantitative trait loci or QTL. Matsuoka and his group [50] were interested in characterizing QTL of major agronomic traits in rice, plant height and grain number. They exploited a natural allelic difference between two parental lines, indica rice cultivar Habataki (shorter, more grains) and japonica rice cultivar Koshihikari (taller, less grain, premier-taste). Four QTLs were identified for plant height $(P h)$ and five QTLs for increasing grain number $(G n)$. The most effective QTL for plant height Ph1 (30\%) located close to the semi-dwarf 1 gene encoding gibberellin 20 oxidase on the long arm of chromosome 1 . Shorter Habataki had a 383 bp deletion in the semi-dwarf 1 gene essentially inactivating the gene activity. The most effective QTL for increasing grain number, Gn1 (44\%) located on the short arm of chromosome 1. They produced Nearly Isogenic Lince (NILs) carrying only one target QTL Gn1 consisting of two loci, QTL-Gn1a and QTL-Gn1b. High resolution mapping of Gn1a-semidormant locates to the $6.3 \mathrm{~kb}$ region between the markers 3A28 and 3A20. One of two open reading frames in this $6.3 \mathrm{kbp}$ region was identified as cytokinin oxidase/dehydrogenese (OsCKX2).

The OsCKX2 gene consists of four exons and three introns with the open reading frame of 565 or 563 amino acid residues. High-yielding rice variety 5150 with more than 400 grains has 11-bp deletion in exon 3 creating a premature termination codon with null phenotype for OsCKX2. The expression profile of OsCKX2 was studied by RT-Southern analysis in place of RT-PCR because of the low expression levels.OsCKX2 was expressed in leaves, culms, inflorescence meristems and flowers, and not detectable in root and embryo. The abundant expression of OsCKX2 in the vascular tissue of developing culm suggests an important role in cytokinin transport to the inflorescence meristem. The highest expression of OsCKX2 in inflorescence meristem was found in the cultivar Koshihikari, the lower in Habataki, and no expression in 5150. The CKX2 promoter: GUS analysis showed the GUS expression in the vascular tissue in developing culms, inflorescence meristems and young flowers. Thus, the higher grain number of Habataki and 5150 might be accounted for by the extremely lower expression level of OsCKX2 in the inflorescence than Koshihikari.

The rice genome has eleven putative CKX genes from OsCKX1 to OsCKX11 [9]. The expression levels of OsCKX1, 6, 7, 8 and 10 were almost not detectable in inflorescence. There were essentially similar expression levels of the remaining OsCKX genes except for OsCKX2 in inflorescence of four cultivars Koshihikari, Habataki, NIL-Gn1a and 5150. The expression level of OsCKX4, 5 and 11 were higher, of OsCKX9 moderate, and ofOsCKX3 lower. No detectable difference was observed in the concentration of cytokinin species, i.e. transzeatin, $i^{6}$ Ade, their ribosides and ribotides among the four cultivars. However, the recent analysis showed ciszeatin is the major cytokinin type in rice, and $\mathrm{N}$ - and O-glucosides are the dominant cytokinin modifications [17]. Thus, the cytokinin content of the above four cultivars Koshihikari, Habataki, NIL-Gn1a and 5150 should be re-tested using the new analytical procedures of Kojima et al. [17].

The expression of $O s C K X 2$ is found to be under control of one upstream dominant negative regulators, Drought and Salt Tolerance (DST) along with potential regulator DENSE AND ERECT PANICLE 1 (DEP1). DST is a zinc-finger transcription factor for abiotic stress drought and salt tolerance involving the control of opening or closure of leaf stomatal aperture [51]. DST also is shown to be a dominant negative regulator on the expression of OsCKX2 gene [52]. DST has two motifs for ethylene-responsive-element-binding factor-associated amphiphilic repression (EAR) at the amino-terminal (DLSLTLAP) and carboxyl-terminal (LDLNLS) of coding sequence. Transactivation activity assay in yeast demonstrated that the deletion of the C-terminal EAR motif, but not of the N-terminal motif abolished the transactivation activity of DSTreg1 mutant. The zinc-finger domain is required for binding of DST protein to two TGNTANN (A/T)T motifs in the 5'-upstream region of OsCKX2 gene, and possibly other OsCKX genes. Loss of C-terminal EAR motif in DSTreg1 mutant also resulted in the reduction of transient transcription activation activity assay in tobacco. It is interesting to note that plants tend to shift from the vegetative growth phase to a reproductive phase setting flowers and seeds to preserve species, when encountering the abiotic stresses such as drought and salt tolerance. It makes an evolutionary sense that one zinc-finger transcription factor DST involves in the regulation of the drought and salt tolerance by closing leaf stomatal opening, and also in the regulation of increased seed yield by using the control of bioactive cytokinin, a major plant growth hormone. 
DEP1 locus confers the two agronomic traits desirable in rice bleeding, the architecture of DENSE AND ERECT PANICLE and the increased grain yield [53]. DEP1 encodes a phosphatidylethanolamine-binding protein. Dominant gain-of-function mutation of DEP1 is caused by 625 bp-deletion resulting in the truncation of carboxyl terminal of this protein. The dominant mutation in dep1 enhances meristematic activity resulting in a reduced length of the inflorescence internode, a doubled number of grains per panicle, and $40 \%$ increase in grain yield per plant. The authors suggested that the increased grain yield might acts through the determination of panicle architecture [53]. It is possible that DEP1 might act as an upstream dominant negative regulator on the expression of OsCKX2 gene [54]. However the direct evidence for the reduced expression of OsCKX2 in the dep1 mutant is not available to our knowledge.

(c) Cytokinin Phosphoribosyl Hydrolase

Lonely Guy $(L O G)$ is the phenotype of rice mutant characterized by loss of female flower organ with a few male flower organs [43]. The loss-of-function mutation of LOG has distinct phenotype in the apical meristem, inflorescence meristem and floral meristems due to premature termination of the shoot meristem activities resulting in the severely reduced panicle size, abnormal branching pattern, and the reduced number of floral organs. A weak phenotype of $\log -1$ (splicing site GT to AT conversion) and log-4 mutants (G73D substitution) flower has only one stamen and no pistil. A stronger phenotype of $\log -5$ (2 bp deletion in exon 1 ) and $\log -6$ (32-bp deletion in exon5) has only a pair of empty glumes. LOG encodes a cytokinin-specific phosphoribosyl hydrolase that converts the less bioactive cytokinin nucleoside 5'-monophosphate to more bioactive cytokinin bases by a single-step activation process of phosphoribosyl hydrolase reaction. The LOG gene encodes a polypeptide of 242 amino acid residues with seven exons and six introns. The rice genome contains 10 LOG-like genes. Among the Arabidopsis 11 genes are seven functional LOG genes in AtLOG1 to LOG5 and LOG7 and LOG8 [55]. The LOG homologues are located adjacent to the adenosine phosphate isopentenyl transferase in $A$. rhizogenes and Rhodococcus fascians (fas ORF6).

The spatial distribution of LOG mRNA is restricted to a subset of cells of shoot apical and axillary meristem, and primary panicle branch meristem and floral meristem in developing panicle [43]. This includes the entire region of presumptive stem cells. The expression pattern of LOG gene suggests a major role for spatial patterns of cytokinin interconversion in the regulation of meristem activity. LOG expression in rice, and AtLOG7 in Arabidopsis is restricted to a group of cells at the shoot apical meristem apex.

(d) Cytokinin Riboside Phosphorylase is a Cytokinin-Binding protein (CKP) in Potato Tubers

A cytokinin-binding protein or StCKP was isolated from potato stolon at the tuber formation stage and found accumulated in dormant potato tuber [44]. The StCKP protein has the sequence similarity to the bacterial purine nucleotidase, nucleosidase and riboside phosphorylase. The StCKP was characterized as a cytokinin-specific riboside phosphorylase with over 100-fold more affinity to trans-zeatin (20 nM K $\mathrm{M}_{\mathrm{M}}$ for zeatin, vs $3.3 \mathrm{mM}$ for adenine). The enzyme mediates a reversible reaction between the bioactive form of cytokinin base (with ribose-1-phosphate) and the less bioactive form of cytokinin riboside (with inorganic phosphate). StCKP can increase the concentration of bioactive cytokinin base in apical shoot meristem thus promoting shoot elongation while StCKP can reduce the concentration of bioactive cytokinin by facilitating the formation of less bioactive storage-form cytokinin riboside in dormant tissue.

The expression of the StCKP gene in potato is under developmental control in a temporal- and spatial-specific manner. StCKP is expressed in the tips of shoot, roots and stolons, and at increased levels in stolon tips at tuber formation and in the periderm of dormant tubers. A low level of expression is detectable in leaves and flowers. The transcripts are more abundant at the tuber formation stage of stolon tips than the proceeding two stages, pre-tuber formation and initial tuber formation stages. Hanke and his group proposed a central role of StCKP in potato tuber formation and dormancy [44]. In elongating stolons, cytokinin ribonucleotides synthesized by IPT are converted to the bioactive form of cytokinin free base by the enzyme cytokinin phosphoribosyl hydrolase (LOG). After stolons stop elongation and transit to tuber formation, the inactive stolon apical meristem will not synthesize cytokinin ribonucleotides, and the transported cytokinin riboside are used to release the bioactive cytokinin free base by CKP enzymes.

\section{Concluding Remarks}

The most fundamental questions in the cytokinin research field are ones concerning the prevalence of cis-zeatin in rice, maize, legumes and other crop plants. A hypothesis is presented that cis-zeatin is synthesized by the 
cis-specific hydroxylation of the terminal methyl group of $\mathrm{N}^{6}$-isopentenyl side chain of $\mathrm{i}^{6}$ Ado or of their mono-, di-, or tri-phosphates catalyzed by the cis-specific hydroxylase [36]. A second potential pathway is the isomerization of trans-zeatin to cis-zeatin by zeatin cis-/trans-isomerase [37]. A second fundamental question to be addressed is the physiological role of cis-zeatin. Trans-Zeatin has been found to be a 100 -fold more active cytokinin in tobacco bioassay than cis-zeatin [2]. Cis-Zeatin might be physiologically as active as trans-zeatin in monocotyledonous plants (rice and maize) and in dicotyledonous legumes (pea, chickpea) and potato. Some have argued for a special function of cis-zeatin to account for the prevalence of the cis-zeatin in a number of plants.

\section{Acknowledgements}

The author acknowledges the financial support in part by the Department of Plant Pathology and Crop Physiology, College of Agriculture, Louisiana State University, and by the Louisiana Agricultural Experiment Station of LSU Agricultural Center.

\section{References}

[1] Mok, M.C. (1994) Cytokinin and Plant Development-An Overview. In: Mok, D.W.S. and Mokeds, M.C., Eds., Cytokinin: Chemistry, Activity and Function, CRC Press, Boca Raton, 155-166.

[2] Skoog, F. (1994) A Personal History of Cytokinin and Plant Hormone Research. In: Mok, D.W.S. and Mokeds, M.C., Eds., Cytokinin: Chemistry, Activity and Function, CRC Press, Boca Raton, 1-14.

[3] Werner, T., Motyka, V., Strnd, M. and Schmulling, T. (2001) Regulation of Plant Growth by Cytokinin. Proceedings of the National Academy of Science of the United States of America, 98, 10487-10492. http://dx.doi.org/10.1073/pnas.171304098

[4] Werner, T., Motyka, V., Laucou, V., Smets, R., Van Onckelen, H. and Schmulling, T. (2003) Cytokinin-Deficient Transgenic Arabidopsis Plants Showed Multiple Developmental Alterations Indicating Opposite Functions of Cytokinins in the Regulation of Shoot and Root Meristem Activity. Plant Cell, 15, 2532-2550. http://dx.doi.org/10.1105/tpc.014928

[5] Werner, T., Nehnevajova, E., Kollmer, I., Novak, O., Strnd, M., Kramer, U. and Schmulling, T. (2010) Root-Specific Reduction of Cytokinin Causes Enhanced Root Growth, Drought Tolerance, and Leaf Mineral Enrichment in Arabidopsis and Tobacco. Plant Cell, 22, 3905-3920. http://dx.doi.org/10.1105/tpc.109.072694

[6] Kyozuka, J. (2009) Control of Shoot and Root Meristem Function by Cytokinin. Current Opinion in Plant Biology, 10, 442-446. http://dx.doi.org/10.1016/j.pbi.2007.08.010

[7] Kyozuka, J., Tokunaga, H. and Yoshida, A. (2014) Control of Grass Inflorescence Form by the Fine-Tuning of Meristem Phase Change. Current Opinion in Plant Biology, 17, 110-115. http://dx.doi.org/10.1016/j.pbi.2013.11.010

[8] Holt, A.L., van Haperen, J.M.A., Groot, E.P. and Laux, T. (2014) Signaling in Shoot and Flower Meristems of Arabidopsis thaliana. Current Opinion in Plant Biology, 17, 96-102. http://dx.doi.org/10.1016/j.pbi.2013.11.011

[9] Sakamoto, T., Sakakibara, H., Kojima, M., Yamamaoto, Y., Nagasaki, H., Inukai, Y., Sato, Y. and Matsuoka, M. (2006) Ectopic Expression of KNOTTED-Like Homeobox Protein Induces Expression of Cytokinin Biosynthesis Genes in Rice. Plant Physiology, 142, 54-62. http://dx.doi.org/10.1104/pp.106.085811

[10] Ikeda, K., Nagasawa, N. and Nagato, Y. (2005) ABERRANT PANICLE ORGANIZATION 1 Temporally Regulates Meristem Identity in Rice. Developmental Biology, 285, 349-360. http://dx.doi.org/10.1016/j.ydbio.2005.03.016

[11] Ikeda-Kawakatsu, K., Maekawa, M., Izawa, T., Itoh, J. and Nagato, Y. (2005) ABBERANT PANICLE ORGANIZATION 2/RFL, the Rice Ortholog of Arabidopsis LEAFY, Suppress the Transition from Inflorescence Meristem to Floral Meristem through Interaction with APO1. Plant Journal, 69, 168-180.

http://dx.doi.org/10.1111/j.1365-313X.2011.04781.X

[12] Kobayashi, K., Yasuno, N., Sato, Y., Yoda, M., Yamazaki, R., Kimizu, M., Yoshida, H., Naganuma, Y. and Kyozuka, J. (2012) Inflorescence Meristem Identity in Rice Is Specified by Overlapping Functions of three AP-1/FUL-Like MADS Box Genes and PAP2, a SEPALLATA MADS Box Gene. Plant Cell, 24, 1848-1859. http://dx.doi.org/10.1105/tpc.112.097105

[13] Liu, C., Teo, Z.W., Bi, Y., Song, S., Xi, W., Yang, X., Yin, Z. and Yu, H. (2013) A Conserved Genetic Pathway Determines Inflorescence Architecture in Arabidopsis and Rice. Developmental Cell, 24, 612-622. http://dx.doi.org/10.1016/j.devcel.2013.02.013

[14] Mok, D.W.S. and Mokeds, M.C. (1994) Cytokinins: Chemistry, Activity, and Function. CRC Press, Inc., Boca Raton.

[15] Mok, D.W.S. and Mok, D.C. (2001) Cytokinin Metabolism and Action. Annual Review of Plant Physiology and Plant 
Molecular Biology, 52, 89-118. http://dx.doi.org/10.1146/annurev.arplant.52.1.89

[16] Sakakibara, H. (2006) Cytokinins: Activity, Biosynthesis and Translocation. Annual Review of Plant Biology, 57, 431449. http://dx.doi.org/10.1146/annurev.arplant.57.032905.105231

[17] Kojima, M., Kamada-Nobusada, T., Komatsu, H., Takei, K., Kuroha, T., Mizutani, M., Ashikari, M., Ueguchi-Tanaka, M., Matsuoka, M., Suzuki, K. and Sakakibara, H. (2012) Highly Sensitive and High-Throughput Analysis of Plant Hormones Using MS-Probe Modification and Liquid Chromatography-Tandem Mass Spectrometry: An Application for Hormone Profiling in Oryza sativa. Plant \& Cell Physiology, 50, 1201-1214. http://dx.doi.org/10.1093/pcp/pcp057

[18] Murai, N., Armstrong, D.J. and Skoog, F. (1975) Incorporation of Mevalonic Acid into Ribosylzeatin in Tobacco Callus RNA Preparations. Plant Physiology, 55, 853-858. http://dx.doi.org/10.1104/pp.55.5.853

[19] Murai, N., Skoog, F., Doyle, M.E. and Hanson, R.S. (1980) Relationship between Cytokinin Production, Presence of Plasmids, and Fasciation Caused by Strains of Corynebacterium fascians. Proceedings of the National Academy of Science of the United States of America, 77, 619-623.

[20] Kasahara, H., Takei, K., Ueda, N., Hishiyama, S., Yamaya, T., Kamiya, Y., Yamaguchi, S. and Sakakibara, H. (2004) Distinct Isoprenoid Origins of cis- and trans-Zeatinbiosyntheses in Arabidopsis. Journal of Biological Chemistry, 279, 14049-14054. http://dx.doi.org/10.1074/jbc.M314195200

[21] Miyawaki, K., Tarkowski, P., Matsumoto-Kitano, M., Kato, T., Sato, S., Tarkowska, D., Tabata, S., Sandberg, G. and Kakimoto, T. (2006) Roles of Arabidopsis ATP/ADP Isopentenyltransferases and tRNAiso Pentynyltrasferases in Cytokinin Biosynthesis. Proceedings of the National Academy of Science of the United States of America, 103, 1659816603. http://dx.doi.org/10.1073/pnas.0603522103

[22] Hashizume, T., Suye, S. and Sugiyama, T. (1982) Isolation and Identification of cis-Zeatin Riboside from Tubers of Sweet Potato (Ipomoea batatas L.). Agricultural and Biological Chemistry, 46, 663-665. http://dx.doi.org/10.1271/bbb1961.46.663

[23] Nicander, B., Bjorkman, P.O. and Tillberg, E. (1995) Identification of an N-Glucoside of cis-Zeatin from Potato Tuber Sprouts. Plant Physiology, 109, 513-516.

[24] Emery, R.J.N., Leport, L., Barton, J.E., Turner, N.C. and Atkin, C.A. (2008) cis-Isomers of Cytokinins Predominate in Chickpea Seeds throughout Their Development. Plant Physiology, 117, 1515-1523. http://dx.doi.org/10.1104/pp.117.4.1515

[25] Quesnelle, P.E. and Emery, R.J.N. (2007) cis-Cytokinins That Predominate in Pisum sativum during Early Embryogenesis will Accelerate Embryo Growth in Vitro. Canadian Journal of Botany, 85, 91-103. http://dx.doi.org/10.1139/b06-149

[26] Stirk, W.A., Novák, O., Václaviková, K., Tarkowski, P., Strnad, M. and van Staden, J. (2008) Spatial and Temporal Changes in Endogenous Cytokinins in Developing Pea Roots. Planta, 227, 1279-1289. http://dx.doi.org/10.1007/s00425-008-0699-z

[27] Martin, R.C., Mok, M.C., Habben, J.E. and Mok, D.W.S. (2001) A Maize Cytokinin Gene Encoding an O-Glucosyltransferase Specific to cis-Zeatin. Proceedings of the National Academy of Science of the United States of America, 98, 5922-5926. http://dx.doi.org/10.1073/pnas.101128798

[28] Veach, Y.K., Martin, R.C., Mok, D.W.S., Malbeck, J., Vankova, R. and Mok, M.C. (2003) O-Glycosylation of cisZeatin in Maize. Characterization of Genes, Enzymes, and Endogenous Cytokinins. Plant Physioogy, 131, $1374-1380$. http://dx.doi.org/10.1104/pp.017210

[29] Yonekura-Sakakibara, K., Kojima, M., Yamaya, T. and Sakakibara, H. (2004) Molecular Characterization of Cytokinin-Responsive Histidine Kinases in Maize. Differential Ligand Preferences and Response to cis-Zeatin. Plant Physiology, 134, 1654-1651. http://dx.doi.org/10.1104/pp.103.037176

[30] Gajdošová, S., Spíchal, L., Kamínek, M., Hoyerová, K., Novák, O., Dobrev, P.I., Galuszka, P., Klíma, P., Gaudinová, A., Žižková, E. Hanuš, J., Dančák, M., Trávniček, B., Peš ek, B., Krupicka, M., Vanková, R., Strnad, M. and Motyka, V. (2011) Distribution, Biological Activities, Metabolism, and the Conceivable Function of cis-Zeatin-Type Cytokinins in Plants. Journal of Experimental Botany, 62, 2827-2840. http://dx.doi.org/10.1093/jxb/erq457

[31] Leonard, N.J., Hecht, S.M., Skoog, F. and Schmitz, R.Y. (1969) Cytokinins: Synthesis, Mass Spectra, and Biological Activity of Compounds Related to Zeatin. Proceedings of the National Academy of Science of the United States of America, 63, 175-182. http://dx.doi.org/10.1073/pnas.63.1.175

[32] Mok, M.C., Mok, D.W.C. and Armstrong, D.J. (1978) Differential Cytokinin Structure-Activity Relationships in Phaseolus. Plant Physiology, 61, 72-75. http://dx.doi.org/10.1104/pp.61.1.72

[33] Kudo, T., Makita, N., Kojima, M., Tokunaga, H. and Sakakibara, H. (2012) Cytokinin Activity of cis-Zeatin and Phenotypic Alteration Induced by Overexpression of Putative cis-Zeatin-O-Glucosyltransferase in Rice. Plant Physiology, 160, 319-331. http://dx.doi.org/10.1104/pp.112.196733 
[34] Yamada, H., Suzuki, T., Terada, K., Takei, K., Ishikawa, K., Miwa, K., Yamashino, T. and Mizuno, T. (2001) The Arabidopsis AHK4 Histidine Kinase Is a Cytokinin-Binding Receptor That Transduces Cytokinin Signals across the Membrane. Plant \& Cell Physiology, 42, 1017-1023.

[35] Rodo, A.P., Brugiere, N., Vankova, R., Malbeck, J., Olson, J.M., Haines, S.C., Martin, R.C., Habben, J.E., Mok, D.W. S. and Mok, M.C. (2008) Over-Expression of a Zeatin O-Glycosylation Gene in Maize Leads to Growth Retardation and Tasselseed Formation. Journal of Experimental Botany, 59, 673-2686.

[36] Takei, K., Yamaya, T. and Sakakibara, H. (2004) Arabidopsis CYP35A1 and CYP735A2 Encode Cytokinin Hydrolases That Catalyze the Biosynthesis of trans-Zeatin. Journal of Biological Chemistry, 279, 41866-41872. http://dx.doi.org/10.1074/jbc.M406337200

[37] Bassil, N.V., Mok, D.W. and Mok, M.C. (1993) Partial Purification of a cis-trans-Isomerase of Zeatin from Immature Seed of Phaseolusvulagaris L. Plant Physiology, 102, 867-872.

[38] Takei, K., Sakakibara, H. and Sugiyama, T. (2001) Identification of Genes Encoding Adenylate Isopentenyltransferase, a Cytokinin Biosynthesis Enzymes, in Arabidopsis thaliana. Journal of Biological Chemistry, 276, 26405-26410. http://dx.doi.org/10.1074/jbc.M102130200

[39] Kakimoto, T. (2001) Identification of Plant Cytokinin Biosynthetic Enzymes as Dimethylallyl Diphosphate: ATP/ADP Isopentenyltransferase. Plant \& Cell Physiology, 42, 677-685. http://dx.doi.org/10.1093/pcp/pce112

[40] Miyawaki, K., Matsumoto-Kitano, M. and Kakimoto, T. (2004) Expression of Cytokinin Biosynthetic Isopentenyltransferase Genes in Arabidopsis: Tissue Specificity and Regulation by Auxin, Cytokinin and Nitrate. The Plant Journal, 37, 128-138. http://dx.doi.org/10.1046/j.1365-313X.2003.01945.X

[41] Brugierè, N., Humbert, S., Rizzo, N., Bohn, J. and Habben, J.E. (2008) A Member of the Maize Isopentenyl Transferase Gene Familly, Zea mays isopentenyl transferase 2 (ZmIPT2), Encodes a Cytokinin Biosyntetic Enzyme Expressed during Kernel Development. Plant Molecular Biology, 67, 215-229. http://dx.doi.org/10.1007/s11103-008-9312-x

[42] Mok, D.W.S. and Martin, R.C. (1994) Cytokinin Metabolic Enzymes. In: Mok, D.W.S. and Mok, M.C., Eds., Cytokinin: Chemistry, Activity and Function, CRC Press, Boca Raton, 129-138.

[43] Kurakawa, T., Ueda, N., Maekawa, M., Kobayashi, K., Kojima, M., Nagato, Y., Sakakibara, H. and Kyozuka, J. (2007) Direct Control of Shoot Meristem Activity by a Cytokinin-Activating Enzyme. Nature, 445, 652-655. http://dx.doi.org/10.1038/nature05504

[44] Hanke, D.E. (2009) Cytokini Nriboside Phosphorylase from Potato and Its Use. WIPO Patent Application WO/2009/ 095715 (GB2009/050081).

[45] Armstrong, D.J. (1994) Cytokinin Oxidase and Regulation of Cytokinin Degradation. In: Mok, D.W.S. and Mok, M.C., Eds., Cytokinin: Chemistry, Activity and Function, CRC Press, Boca Raton, 139-154.

[46] Bilyeu, K., Cole, J.L., Laskey, J.G., Riekhof, W.R., Esparza, T.J., Krammer, M.D. and Morris, R.O. (2001) Molecular and Biochemical Characterization of a Cytokinin Oxidase from Maize. Plant Physiology, 125, 378-386. http://dx.doi.org/10.1104/pp.125.1.378

[47] Bartrina, I., Otto, E., Strnad, M., Werner, T. and Schmülling, T. (2011) Cytokinin Regulates the Activity of Reproductive Meristems, Flower Organ Size, Ovule Formation, and Thus Seed Yield in Arabidopsis thaliana. Plant Cell, 23, 6980. http://dx.doi.org/10.1105/tpc.110.079079

[48] Werner, T. and Schummülling, T. (2009) Cytokinin Action in Plant Development. Current Opinion in Plant Biology, 12, 527-538. http://dx.doi.org/10.1016/j.pbi.2009.07.002

[49] Schmulling, T., Werner, T., Riefler, M., Krupkova, E. and Manns, I.B. (2003) Structure and Function of Cytokinin Oxidase/Dehydrogenase Gene of Maize, Rice, Arabidopsis and Other Species. Journal of Plant Research, 116, 241252. http://dx.doi.org/10.1007/s10265-003-0096-4

[50] Ashikari, M., Sakakibara, H., Lin, S., Yamamoto, T., Takashi, T., Nishimura, A., Angeles, E.R., Qian, Q., Kitano, H. and Matsuoka, M. (2005) Cytokinin Oxidase Regulates Rice Grain Production. Science, 309, 741-745. http://dx.doi.org/10.1126/science.1113373

[51] Huang, X.Y., Chao, D.Y., Gao, J.P., Zhu, M.Z., Shi, M. and Lin, H.X. (2009) A Previously Unknown Zinc Finger Protein, DST, Regulates Drought and Salt Tolerance in Rice via Stomatal Aperture Control. Genes \& Development, 23, 1805-1817. http://dx.doi.org/10.1101/gad.1812409

[52] Li, S., Zao, B., Yuan, D., Duan, M., Qian, Q., Tang, L., Wang, B., Liu, X., Zhnag, J., Wang, J., Sun, J., Liu, Z., Feng, Y.Q., Yuan, L. and Lin, C. (2013) Rice Zinc Finger Protein DST Enhances Grain Production through Controlling Gn1a/OsCKX2 Expression. Proceedings of the National Academy of Science of the United States of America, 110, 3167-3172.

[53] Huang, X.Z., Qian, Q., Liu, Z., Sun, H., He, S., Luo, D., Xia, G., Chu, C., Li, J. and Fu, X. (2009) Natural Variation at the DEP1 Locus Enhances Grain Yield in Rice. Nature Genetics, 41, 494-497. http://dx.doi.org/10.1038/ng.352 
[54] Sreenivasulu, N. and Schnurbusch, T. (2011) A Genetic Playground for Enhancing Grain Number in Cereals. Trends in Plant Science, 17, 91-101. http://dx.doi.org/10.1016/j.tplants.2011.11.003

[55] Kuroha, T., Tokunaga, H., Kojima, M., Ueda, N., Ishida, T., Nagawa, S., Fukuda, H., Sugimoto, K. and Sakakibara, H. (2009) Functional Analyses of LONELY GUY Cytokinin-Activation Enzymes Reveal the Importance of the Direct Activation Pathway in Arabidopsis. The Plant Cell, 21, 3152-3169. http://dx.doi.org/10.1105/tpc.109.068676 
Scientific Research Publishing (SCIRP) is one of the largest Open Access journal publishers. It is currently publishing more than 200 open access, online, peer-reviewed journals covering a wide range of academic disciplines. SCIRP serves the worldwide academic communities and contributes to the progress and application of science with its publication.

Other selected journals from SCIRP are listed as below. Submit your manuscript to us via either submit@scirp.org or Online Submission Portal.
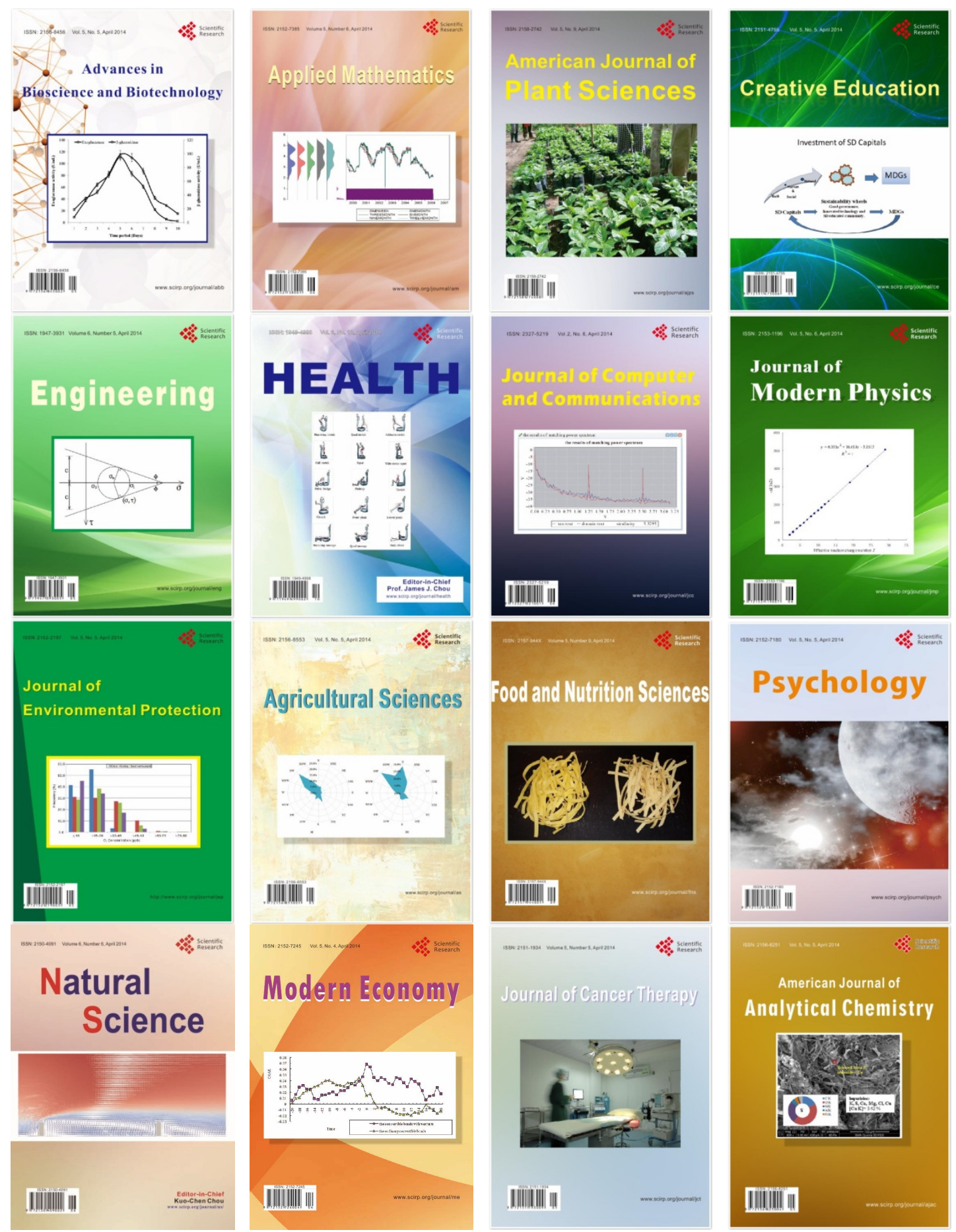